\title{
Der Tarifkonflikt bei der Deutschen Telekom AG
}

Lothar Schröder

Im Konflikt zwischen der Vereinten Dienstleistungsgewerkschaft (ver.di) und der Deutschen Telekom AG (DTAG) um die konzerninterne Auslagerung von drei Servicegesellschaften („T-Service“) konnte nach elf Wochen Warn- und Erzwingungsstreiks mit insgesamt rund $450.000 \mathrm{Ar}$ beitskampftagen eine Einigung erzielt werden. Die Medien verkürzten das fast siebzigseitige Verhandlungsergebnis vom 20. Juni 2007 auf eine sehr schlichte Frage: Wurde für den DTAG-Vorstand ein Sieg, für ver.di eine glatte Niederlage vereinbart? ${ }^{1}$ Eine solche Bilanz löste bei den Streikenden, die diese Auseinandersetzung mit imponierendem Engagement und gegen massive Pressionen des Arbeitgebers geführt hatten, Unverständnis aus. Sie schätzten den erreichten Kompromiss offensichtlich mehrheitlich anders ein als die veröffentlichte Meinung. Nur so lässt sich die Tatsache erklären, dass sich an der abschließenden Urabstimmung 86,5 \% der betroffenen ver.di-Mitglieder beteiligten, von denen 72,6\% dem Tarifergebnis zustimmten.

Nicht nur angesichts einer verkürzten, zum Teil auch verzerrenden Medienberichterstattung, sondern auch aufgrund der über die Telekom hinausreichenden Bedeutung dieser Auseinandersetzung empfiehlt sich ein genauerer Blick auf deren Resultate. Dazu ist es hilfreich, noch einmal die ursprünglichen Intentionen der Konfliktbeteiligten zu rekapitulieren.

\section{DIE ZIELE DER TELEKOM}

Was waren die Ziele der DTAG, die diese Auseinandersetzung in der aggressiven Angreiferrolle und in bis dato ungekannter Härte, ja Rücksichtslosigkeit, geführt hat? Rein formell verfolgte das Telekom-Management den Plan, rund 50.000 Beschäftigte der Festnetzsparte in drei Gesellschaften auszugliedern, die sich um den technischen Service, die Kundenbetreuung in Call-Centern und den Bau und Betrieb der Netzinfrastruktur kümmern sollten. Restriktionen irgendwelcher Art, die eine rasche Veräußerung dieser zunächst konzerneigenen Einheiten behindert hätten, waren dabei nicht vorgesehen. Materiell verband sich dieser Schritt mit der Absicht, durch eine erhebliche Verschlechterung der Konditionen für die betroffenen Beschäftigten die wirtschaftliche Situation des Konzerns in einem Konkurrenzumfeld zu verbessern, das - so ein wiederholt von Arbeitgeberseite genannter „Benchmark“um 30 bis $50 \%$ unter dem Telekom-Lohnniveau liege.

Konkret ging es dem DTAG-Management u.a. um

- eine Absenkung der nominalen Entgelte um $12 \%$,

- einen Ausschluss von Entgelterhöhungen bis zum Jahr 2011,

- eine drastische Reduzierung der Löhne neu einzustellender Beschäftigter bis herunter auf ein Dumpingniveau von wenig mehr als $7 €$ pro Stunde

- eine Verlängerung der Wochenarbeitszeit von 34 auf bis zu 41 Stunden für einzelne Beschäftigtengruppen (Beamte/Beamtinnen).

Im Kern zielte das radikale Kostensenkungsprogramm des Telekom-Managements darauf ab, mit einer Organisationsänderung aus einem über Jahrzehnte gewachsenen und erkämpften tarifvertraglichen Gefüge auszusteigen. Dieser Kahlschlag sollte nicht nur mit erheblichen materiellen Einbußen für die Beschäftigten einhergehen, sondern auch mit einem Abbau von Sicherheit, wurde doch der bislang bestehende Schutz vor betriebsbedingten Entlassungen ausdrücklich zur Disposition gestellt.

\section{DIE ZIELE VON VER.DI}

Wie sahen angesichts dieses Generalangriffs der Arbeitgeberseite die Verteidigungslinien von ver.di aus? Von Bedeutung ist zunächst die Klarstellung, was realistischerweise nicht tarif- und arbeitskampftaugliches Ziel sein konnte: Zwar hielt ver.di die Ausgliederung der drei Servicegesellschaften aus vielerlei Gründen für unternehmenspolitisch falsch und positionierte sich mit großem Nachdruck gegen diese Maßnahme - u. a. bei der Abstimmung im Aufsichtsrat, die von einer Demonstration von 13.000 Gewerkschafterinnen und Gewerkschaftern vor der Bonner Telekom-Zentrale begleitet wurde. Nachdem die Ausgründungsentscheidung aber gegen das ausdrückliche gewerkschaftliche Votum gefallen war, hatte ver.di keine Handhabe mehr, diese in der Dispositionsgewalt des Vorstands liegende Organisationsmaßnahme zu verhindern. Deshalb konnte dies auch weder Tarifvertragsforderung noch Streikziel sein.

Oberste Maxime von ver.di war es vielmehr, den unmittelbar betroffenen Beschäftigten für den Fall der Ausgründung ein Höchstmaß an Schutz und Sicherheit zu erhalten und die vom DTAG-Management vorgesehenen Einschnitte abzuwehren, zumindest aber in Grenzen zu halten. Dem diente zum einen die Forderung nach einem Sozialtarifvertrag „Auslagerungsschutz“, zum anderen das Ziel, die neuen Einheiten auf möglichst hohem Niveau voll zu tarifieren. Materiell ging es, um nur die wichtigsten Punkte anzuführen, darum

- eine Kürzung der bestehenden Einkommen - also einen „Griff in den Geldbeutel“ der vorhandenen Beschäftigten - zu verhindern;

- die Entgelte für neu einzustellende Beschäftigte der ausgegliederten Gesellschaften nicht auf Dumpingniveau absinken zu lassen;

\footnotetext{
1 Differenziertere Töne wurden erst nach einigen Tagen und nur in wenigen Pressebeiträgen angeschlagen. Die "Tageszeitung“ beispielsweise, die in ihrer ersten Reaktion am 21. Juni 2007 noch mit dem medialen Mainstream verkündet hatte, die Unternehmensseite habe "sich im Telekom-Streik weitgehend durchgesetzt ${ }^{\prime \prime}$, relativierte ihre Sicht der Dinge am 25. Juni 2007: „Die Details der Einigung werden erst jetzt bekannt. Offenbar fällt sie für die Beschäftigten günstiger aus als zunächst berichtet wurde."
}

Lothar Schröder, Mitglied des ver.di-Bundesvorstandes, Leiter des Fachbereichs Telekommunikation, Informationstechnologie, Datenverarbeitung. e-mail: Lothar.Schroeder@verdi.de 
- den Schutz vor betriebsbedingten Beendigungskündigungen für einen möglichst langen Zeitraum zu erhalten;

- einen Schutz vor Verkauf der neuen Einheiten zu vereinbaren;

- eine Verlängerung der Arbeitszeit abzuwehren;

- den Personalabbau zu beenden;

- das gewachsene tarifvertragliche Rahmenwerk der Muttergesellschaft auf die neuen Einheiten zu übertragen.

Es gibt nichts daran zu deuteln: Alle diese Forderungen, so existenziell wichtig sie für die Betroffenen waren, blieben defensiver Natur. Anders als in der Mehrzahl der Tarifauseinandersetzungen des ersten Halbjahres 2007 stand im Telekom-Konflikt von vornherein nicht die Durchsetzung von Reallohnsteigerungen auf der Agenda, sondern die Begrenzung von Verlusten. Die gewerkschaftlichen Ziele waren bescheiden, wenn man traditionelle tarifliche Auseinandersetzungen um Entgeltzuwächse zum Maßstab nimmt und zudem berücksichtigt, dass dieser Konflikt in einer Phase des konjunkturellen Aufschwungs stattfand. Andererseits waren sie ausgesprochen ambitioniert angesichts der Aggressivität, der Entschlossenheit und der rechtlich abgesicherten Handlungs- und Erpressungsmöglichkeiten der Gegenseite.

\section{DAS TARIFERGEBNIS - WEDER TRIUMPH NOCH DEBAKEL}

Diese widersprüchliche, für ver.di naturgemäß wenig komfortable Ausgangskonstellation muss bei der Beurteilung des Tarifergebnisses berücksichtigt werden. Von einem gewerkschaftlichen Triumph kann sicherlich nicht die Rede sein, dazu enthält die letztlich geschlossene Vereinbarung einige zu bittere Pillen. Dass die da und dort genüsslich kolportierte These vom Debakel für ver.di der Realität allerdings noch weit weniger gerecht wird, dürfte sich unvoreingenommenen Zeitgenossen bei näherer Betrachtung alsbald erschließen.

\section{KRITISCHE KOMPONENTEN}

Zunächst zu den unzweifelhaft negativen Komponenten des Abschlusses. Die Arbeitgeberseite vermochte eine Erhöhung der wöchentlichen Arbeitszeit von 34 auf 38 Stunden durchzusetzen, ohne dass hierfür ein Lohnausgleich vereinbart werden konnte. ${ }^{2}$ Geringfügig abgefedert wird die- ser Rückschritt dadurch, dass die Beschäftigten damit nicht vier, sondern "nur“ zweieinhalb unbezahlte Stunden mehr leisten, da die Berechnung des Lohnes bislang auf einer fiktiven 35,5-Stunden-Woche basierte. Auch setzte ver.di im Gegenzug einen tarifvertraglichen Qualifizierungsanspruch in der Größenordnung von einer halben Stunde pro Woche durch - die rechnerische Wochenarbeitszeit beträgt folglich 37,5 Stunden. Die durch die Arbeitszeitverlängerung erweiterten Beschäftigungskapazitäten werden, so die Festlegung im Tarifvertrag, „zur Verringerung von externer Vergabe und externer Leiharbeit verwendet" und somit nicht in Personalabbau umgesetzt. Zur Überwachung dieser Verpflichtung des Arbeitgebers sind Monitoringverfahren unter Beteiligung der Tarifvertragsparteien und der Mitbestimmungsgremien vereinbart worden. Ungeachtet dieser wichtigen Differenzierungen und Relativierungen lässt sich im Ergebnis nicht beschönigen, dass die Arbeitszeitverlängerung ein herber Rückschlag für ver.di und die betroffenen Beschäftigten ist.

Gleichfalls kritisch zu bewerten ist die deutliche Absenkung der Einstiegsentgelte für neu einzustellende Mitarbeiter der drei Servicegesellschaften. Von Dumpinglöhnen, wie sie die DTAG anstrebte, kann allerdings keine Rede sein: Ein neu eingestellter Call-Center-Agent ohne Berufserfahrung wird künftig bei der Deutschen Telekom Kundenservice $\mathrm{GmbH}$ rund $24.000 €$ pro Jahr verdienen - dies liegt im Mittelfeld der in dieser Branche üblichen Einkommen und weit über dem von der Arbeitgeberseite geforderten Jahreswert von ca. $19.000 €$. Hinzu kommt, dass die auf dieser Entgeltbasis Eingestellten zügig an das allgemeine Einkommensniveau in den drei Gesellschaften herangeführt werden - eine „zweite Lohnebene“ wird es für die Newcomer somit nicht geben.

\section{ERFOLGREICHE SICHERUNGEN}

Die größten Irritationen hat verständlicherweise die Tatsache hervorgerufen, dass die Berechnungsbasis der Löhne der in die drei Gesellschaften wechselnden Beschäftigten im Vergleich zum Status quo ante um $6,5 \%$ nach unten angepasst wird. Dieser Sachverhalt bestimmte denn auch zumindest im Anfangsstadium der Berichterstattung den Tenor der Schlagzeilen. Allerdings - und dies gehört bereits zu den positiven Elementen des Tarifabschlusses - büßen die vorhandenen Beschäftigten trotz dieser Absenkung der Berechnungsgrundlagen nominal keinen Cent ein, ihre Einkommen bleiben aufgrund der vereinbarten Schutzregelungen konstant. Die Mechanismen zur Stabilisierung der Einkommen greifen mittelfristig und sind über einen Zeitraum von insgesamt 42 Monaten teuer für die Telekom: In den ersten $18 \mathrm{Mo-}$ naten ab dem 1. Juli 2007 gleicht die Telekom die besagte Kürzung um 6,5 \% vollständig aus, ab dem Jahr 2009 zu zwei Dritteln, ab 2010 dann noch zu einem Drittel. Gelingt es ver.di, in der Tarifbewegung ab Januar 2009 jeweils eine Entgelterhöhung von $2,13 \%$ durchzusetzen, wäre eine Lohnkürzung auf Dauer abgewehrt - ein sicherlich nicht unrealistisches Szenario. Selbst für den Fall, dass ein solcher Anstieg um 2,13\% nicht zu schaffen sein sollte, sieht das jetzige Tarifergebnis Ausgleichszahlungen des Arbeitgebers vor - zu diesem Zweck bildet das Unternehmen eine Rücklage von 18 Mio. €. Die Beschäftigten der ausgegliederten Gesellschaften erleiden keine nominalen Entgelteinbußen, zumal die Ausgangslage für Reallohnsteigerungen in den kommenden Jahren besser zu werden scheint. Das für ver.di zentrale und für die betroffenen Arbeitnehmerinnen und Arbeitnehmer existenzielle Ziel der Einkommenssicherung konnte damit gegen den massiven Druck der Arbeitgeber realisiert werden - ein Resultat, das ohne Arbeitskampf niemals zu erreichen gewesen wäre.

Gleiches gilt für das ebenfalls herausragend bedeutsame Ziel der Arbeitsplatzsicherheit: Der Ausschluss betriebsbedingter Beendigungskündigungen konnte für die Mitarbeiter der Servicegesellschaften vom Zeitpunkt des Tarifabschlusses an gemessen - für fünfeinhalb Jahre bis zum 31.12.2012 vereinbart werden. Dies ermöglicht den Beschäftigten auf mittlere Sicht eine verlässliche Lebensplanung und wertvollen Schutz in Zeiten, die in Anbetracht der ökonomischen und technischen Umbrüche im Telekommunikationssektor weiterhin hoch turbulent bleiben dürften. Der Tarifabschluss sichert jedoch nicht nur die

\footnotetext{
2 Damit hat die Telekom den im „Beschäftigungsbündnis" mit ver.di 2004 eingeschlagenen Kurs der Arbeitszeitverkürzung wieder verlassen. Damals war die Wochenarbeitszeit von 38 auf 34 Stunden zurückgefahren worden, was half, rund 10.000 Arbeitsplätze zu sichern.
} 
Existenzen der bereits Beschäftigten, sondern eröffnet zusätzlich Perspektiven für Jüngere: Im Rahmen einer „Beschäftigungsbrücke" werden in den Jahren 2007 bis 2008 bei der Telekom 4.150 Nachwuchskräfte (auf Vollzeitstellen umgerechnet) eingestellt - unbefristet und nicht in Zeit- oder Leiharbeit. Zudem wird der geplante Personalabbau in den drei Gesellschaften auf Fälle beschränkt, in denen Beschäftigte freiwillig ausscheiden.

Nicht weniger essentiell als die Erhaltung von Einkommens- und Arbeitsplatzsicherheit ist für die Beschäftigten und ver.di, dass es durch die Volltarifierung der Servicegesellschaften gelungen ist, die wesentlichen Tarifverträge der Konzernmutter auf die neuen Einheiten zu übertragen. Damit gelten z. B. die Rationalisierungsschutzbestimmungen in der hohen Qualität der DTAG auch in den drei ausgegliederten GmbHs. Gleiches trifft für viele andere soziale Besitzstände der Beschäftigten, wie die Regelungen zur betrieblichen Altersversorgung oder zur Altersteilzeit, zu. Im Ergebnis wurde so die Absicht des Telekom-Vorstandes durchkreuzt, das komplette Gefüge an kollektivvertraglicher Regulierung mit dem Federstrich einer Organisationsänderung vom Tisch zu wischen.

Nur unter dem Druck des sechswöchigen Erzwingungsstreiks gelang schließlich eine Verpflichtung des Arbeitgebers auf die Zusage, „bis zum 31.12.2010 auf einen Verkauf der Mehrheitsanteile an den drei Servicegesellschaften (,change of control' Kontrollwechsel)“ zu verzichten. Auch für den Fall eines etwaigen Verkaufs nach diesem Zeitpunkt bleibt der Ausschluss betriebsbedingter Beendigungskündigungen bis Ende 2012 vereinbarungsgemäß ebenso erhalten wie die Fortgeltung der für die jeweiligen Gesellschaften bestehenden Tarifverträge.

\section{DREI VORLÄUFIGE EINSCHÄTZUNGEN}

Angesichts der Komplexität des Tarifwerks, von dem hier nur wichtige Eckpfeiler referiert wurden, fällt ein schlichtes Gesamtfazit schwer, zumal wenn es allein mit den Kategorien „Sieg“ oder „Niederlage“ auszukommen versucht. Legt man die eingangs genannten fünf Ziele zugrunde, mit denen ver.di in diese Auseinandersetzung ging - keine Einkommenskürzungen für die vorhandenen Beschäftigten, Sicherung der Arbeitsplätze, keine Dumpinglöhne für Neueingestellte, Fortgeltung der Tarifver- träge, keine Arbeitszeitverlängerung - so ist $\mathrm{zu}$ konstatieren, dass vier von diesen fünf Zielen erreicht werden konnten. Trotz bitterer Zugeständnisse ist es gelungen, gegen den Generalangriff der Arbeitgeberseite Schutz und Sicherheit für die Beschäftigten $\mathrm{zu}$ verteidigen und die Grundlagen tarifvertraglicher Regulierung zu erhalten. Das ist mehr als respektabel, gleichwohl aber kein Anlass für Triumphgeschrei. Wohl aber zur sorgsamen und durchaus auch selbstkritischen Analyse. Dazu abschließend noch drei knappe Einschätzungen vorläufiger Natur:

(1) Ein bemerkenswertes Novum in der Tarifgeschichte der DTAG war zum einen, dass in diesem Konflikt erstmals mit Blackstone ein Finanzinvestor im Hintergrund als „Treiber“ des Unternehmensmanagements agierte. Insoweit war dieser Streik der erste seiner Dimension unter dem neuen Regime des Finanzmarktkapitalismus. Obwohl Blackstone seit April 2006 nur über einen Minderheitsanteil von 4,5\% am Kapital der Telekom verfügt, ist der Einfluss des Private-Equity-Unternehmens auf den Konzern massiv zu spüren. Die Gangart des Telekom-Managements hat sich seit dem Blackstone-Einstieg deutlich verschärft, die traditionell eher sozialpartnerschaftlichen Arbeitsbeziehungen bei der DTAG haben sich erheblich verschlechtert. Der von den übersteigerten Renditeerwartungen des Finanzinvestors ausgehende Druck mündete in diesem Konflikt in einen beispiellosen Verteilungsextremismus der Arbeitgeberseite: Während den Beschäftigten gravierende materielle Einbußen in einer Größenordnung von bis zu 900 Mio. $€$ abverlangt werden sollten, standen für den DTAG-Vorstand weder eine Rekorddividende von 3,2 Mrd. $€$ noch ein Wertschöpfungsziel von 19 Mrd. $€$ für 2007 zur Disposition; diese Rahmendaten wurden vielmehr unter Verweis auf die Forderungen des Kapitalmarkts für sakrosankt erklärt. Im Zusammenwirken mit der globalen Gewerkschaft UNI hat ver.di die fragwürdige Rolle des Finanzinvestors Blackstone in dieser Auseinandersetzung öffentlich thematisiert und dabei auch das Investitionsverhalten gewerkschaftlich beeinflusster Pensionsfonds ins Spiel gebracht - mit bemerkenswerter medialer Resonanz, ${ }^{3}$ wenngleich ohne spürbare Wirkungen auf das Verhalten des DTAGVorstands. Ob es tatsächlich ein erfolgversprechender Ansatz sein kann, „Heu- schrecken" auf solche Art und Weise domestizieren zu wollen, mag dahinstehen. In jedem Fall werden die Gewerkschaften damit zu rechnen und innovative Antworten darauf zu finden haben, dass Finanzinvestoren auch in künftigen Verteilungsauseinandersetzungen de facto mit am Tisch sitzen - der Telekom-Konflikt dürfte hier nur ein Aufgalopp gewesen sein.

(2) Darüber hinaus haben sich bei dieser Auseinandersetzung - für ver.di im Telekomsektor erstmals in dieser Brisanz - gravierende Probleme in der Wirkungsweise des altehrwürdigen $₫ 613$ a BGB offenbart. Dieser entfaltet seine generelle einjährige Schutzwirkung bei einem Betriebsübergang dann nicht, wenn im neuen Unternehmen bereits Tarifverträge existieren, die mit derselben Gewerkschaft geschlossen wurden. Gibt es eine solche Konstellation, „passiert das Gegenteil des erwarteten Schutzes: Die bisherigen Rechte werden unmittelbar und sofort beseitigt, es gilt nur noch der neue Tarifvertrag". ${ }^{4}$ Die DTAG hat sich diesen Passus zunutze gemacht, indem sie die neuen Servicegesellschaften auf Teile von seit Längerem bestehende GmbHs verschmolzen hat, für die ver.di zu früheren Zeiten und unter gänzlich anderen Randbedingungen bereits Tarifverträge mit unterschrieben hatte - mit niedrigerem materiellen Niveau als in der Konzernmutter. Was damals ein begründbarer Schritt gewesen ist, rächte sich nun. Für die Arbeitgeberseite resultierte aus einer solchen Konstellation ein beträchtliches Repertoire an Gestaltungs- und Erpressungsmöglichkeiten, welches die DTAG weidlich ausschöpfte, um die Beschäftigten auch sehr persönlich unter Druck zu setzen. Auch wenn diese Strategie am Ende nicht verfangen hat, so erscheinen doch wenigstens zwei Schlussfolgerungen unabweisbar: Zum einen eine noch größere gewerkschaftliche

\footnotetext{
3 Für die "Süddeutsche Zeitung" (24.04.2007) beispielsweise stand angesichts der Initiative von ver.di und UNI "die Welt ... Kopf: Eine Gewerkschaftsorganisation droht einem Finanzinvestor, ihm Geld zu entziehen, sollte er weiter eine arbeitnehmerfeindliche Politik verfolgen. ... Für die Private-Equity-Branche, die sich seit Jahren mit dem Vorwurf konfrontiert sieht, sich auf Kosten der Arbeitnehmer die Taschen zu füllen, gewinnt die Diskussion um ihre gesellschaftliche Rolle damit eine neue Dimension."

4 Hamm, I./Rupp, R. (2007): Mitbestimmung bei Veräußerung und Restrukturierung, Frankfurt, S. 168
} 
Vorsicht und Zurückhaltung beim Abschluss von Ausnahmetarifverträgen, zum anderen gesetzgeberische Initiativen, die der Pervertierung des Schutzcharakters von $\S 613$ a BGB in der beschriebenen Weise einen Riegel vorschieben. Die Einführung des Günstigkeitsprinzips in den $\S 613$ a könnte helfen.

(3) Und schließlich: Aus gewerkschaftlicher Sicht hoch problematisch wirkte bei diesem Konflikt auch der Tatbestand, dass ver.di im liberalisierten Telekommunikationsmarkt im Kern ihres Organisationsbereichs zunehmend unter den Druck einer
Branchensituation gerät, in der deutlich schlechtere Standards als beim Ex-Monopolisten und prekäre Beschäftigung auf dem Vormarsch sind. Diese Problematik ist im Grunde seit Langem bekannt, hat aber nunmehr eine Brisanz erlangt, die zu Konsequenzen zwingt. Wie diese aussehen müssen, sei abschließend mit zwei Aussagen illustriert. Eine trifft eine interne Streikanalyse eines ver.di-Landesbezirksfachbereichs. Dort wird festgestellt, dass „die Telekom-Beschäftigten massiv unter Druck bleiben, wenn es nicht gelingt, die Tarifstandards in der Branche anzuheben." Und in der „Tageszeitung“ (21.06.2007) bringt die Korrespondentin Ulrike Herrmann die eigentliche Lehre aus diesem Konflikt so auf den Punkt: „Es wird erst einen einheitlichen Flächentarifvertrag geben können, wenn eine deutliche Mehrheit aller Beschäftigten der Telekom-Branche einsieht, dass sie sich dringend organisieren müssen.... Nur dann wird das Spiel enden, dass die Beschäftigten der einzelnen Firmen gegeneinander ausgespielt werden." Die zentrale gewerkschaftliche Gegenwarts- und Zukunftsaufgabe sollte damit hinreichend klar beschrieben sein. 